\title{
CONDIÇÕES DE ATMOSFERA CONTROLADA, TEMPERATURA E UMIDADE RELATIVA NO ARMAZENAMENTO DE MAÇÃS 'FUJI'
}

\author{
Controlled atmosphere, temperature and relative humidity \\ conditions on the storage of 'Fuji' apples
}

\author{
Auri Brackmann ${ }^{1}$, Ricardo Fabiano Hettwer Giehl ${ }^{2}$, Ivan Sestari ${ }^{3}$, Cristiano André Steffens ${ }^{4}$
}

\begin{abstract}
RESUMO
Conduziu-se este trabalho com o objetivo de avaliar o efeito da temperatura de armazenamento, níveis de umidade relativa do ar (UR) e pressões parciais de $\mathrm{O}_{2}$ sobre a qualidade de maçãs 'Fuji' conservadas em atmosfera controlada (AC). O delineamento experimental utilizado foi o inteiramente casualizado com quatro repetições, contendo 25 frutos cada uma. Os tratamentos avaliados foram: armazenamento a $0,5^{\circ} \mathrm{C}$ sob AC com (1) $0,7 \mathrm{kPa}$ de $\mathrm{O}_{2}$ e (2) $1,0 \mathrm{kPa}$ de $\mathrm{O}_{2}$; armazenamento a $-0,5^{\circ} \mathrm{C}$ sob AC com (3) $0,7 \mathrm{kPa}$ de $\mathrm{O}_{2}$; (4) $1,0 \mathrm{kPa}$ de $\mathrm{O}_{2}$; (5) 1,0 kPa de $\mathrm{O}_{2}$ mais baixa UR na câmara; (6) $1,0 \mathrm{kPa}$ de $\mathrm{O}_{2}$, após 2 dias de exposição a 20 ${ }^{\circ} \mathrm{C} \mathrm{e} \mathrm{(7)} 1,0 \mathrm{kPa}$ de $\mathrm{O}_{2}$ após 1 mês de armazenamento refrigerado (AR). Em todos os tratamentos as pressões parciais de $\mathrm{CO}_{2}$ foram mantidas abaixo de $0,5 \mathrm{kPa}$. Os frutos foram expostos a uma UR de $96 \%$, exceto no tratamento com baixa UR, em que os níveis permaneceram próximos a 90\%. De modo geral, as condições de armazenamento avaliadas neste trabalho não proporcionaram diferenças significativas na qualidade de maçãs 'Fuji', após oito meses de armazenamento e exposição a $20^{\circ} \mathrm{C}$ durante sete dias. No entanto, o atraso no resfriamento, por meio da exposição dos frutos a $20^{\circ} \mathrm{C}$ por dois dias antes do armazenamento a $-0,5^{\circ} \mathrm{C}$ sob $\mathrm{AC} \operatorname{com~} 1,0 \mathrm{kPa}$ de $\mathrm{O}_{2}$, e o retardamento na instalação das condições de $\mathrm{AC}\left(1,0 \mathrm{kPa}\right.$ de $\left.\mathrm{O}_{2} \mathrm{a}-0,5^{\circ} \mathrm{C}\right)$ em um mês, apresentaram bons resultados no controle das podridões após sete dias a $20^{\circ} \mathrm{C}$, especialmente quando comparados com o armazenamento a $0,5^{\circ} \mathrm{C}$ sob $\mathrm{AC}$ com $0,7 \mathrm{e} 1,0 \mathrm{kPa}$ de $\mathrm{O}_{2}+<0,5 \mathrm{kPa}$ de $\mathrm{CO}_{2}$.
\end{abstract}

Termos para indexação: Malus domestica Borkh., degenerescência da polpa, distúrbios fisiológicos.

\section{ABSTRACT}

This experiment was carried out with the objective to evaluate the effect of temperature, relative humidity (RH) levels and $\mathrm{O}_{2}$ partial pressures on the quality of 'Fuji' apples stored on controlled atmosphere (CA). The experimental design was entirely randomized with four replications of 25 fruits. Evaluated treatments were: storage at $0.5^{\circ} \mathrm{C}$ under $\mathrm{CA}$ with (1) $0.7 \mathrm{kPa} \mathrm{O}_{2}$ and $(2)$ $1.0 \mathrm{kPa} \mathrm{O}$; storage at $-0.5^{\circ} \mathrm{C}$ under CA with (3) $0.7 \mathrm{kPa} \mathrm{O}_{2}$; (4) $1.0 \mathrm{kPa} \mathrm{O}_{2}$; (5) $1.0 \mathrm{kPa} \mathrm{O}$ plus low $\mathrm{RH}-90 \%$; (6) $1.0 \mathrm{kPa} \mathrm{O}{ }_{2}$ after 2 days at $20^{\circ} \mathrm{C}$ (delayed storage) and (7) $1.0 \mathrm{kPa} \mathrm{O}{ }_{2}$ after 1 month of cold storage. Partial pressures $\mathrm{CO}_{2}$ were maintained lower than $0.5 \mathrm{kPa}$ in all treatments. In general, storage conditions evaluated in this work did not allow significative differences on quality maintenance of 'Fuji' apples after 8 months of storage plus 7 days of shelf-life. However delayed storage, through the placing of fruits at $20^{\circ} \mathrm{C}$ during 2 days before transfer to $\mathrm{CA}$ with $1.0 \mathrm{kPa} \mathrm{O}+<0.5 \mathrm{kPa} \mathrm{CO}_{2}$ at $-0.5^{\circ} \mathrm{C}$, and cold storage of fruits during 1 month before CA storage, exhibited good results on rot control after 7 days of shelf-life at $20^{\circ} \mathrm{C}$, especially when compared with storage at $0.5^{\circ} \mathrm{C}$ under $\mathrm{CA}$ with 0.7 and $1.0 \mathrm{kPa} \mathrm{O}_{2}+<0.5 \mathrm{kPa} \mathrm{CO}_{2}$.

Index terms: Malus domestica Borkh., flesh breakdown, physiological disorders.

Recebido para publicação em 21 de julho de 2004 e aprovado em 4 de março de 2005)

\section{INTRODUÇÃO}

Durante o armazenamento de maçãs 'Fuji' as baixas temperaturas reduzem o metabolismo dos frutos aumentando seu período de conservação. No entanto, abaixo de um nível crítico, podem provocar o colapso das células e a manifestação de distúrbios fisiológicos. De acordo com Little \& Barrand (1989), a maçã 'Fuji' não é suscetível ao dano por baixa temperatura, podendo ser conservada satisfatoriamente sob refrigeração a $-0,5^{\circ} \mathrm{C}$ (BRACKMANN et al., 1998).

$\mathrm{O}$ armazenamento em condições de atmosfera controlada (AC) permite um aumento ainda maior da vida pós-colheita da maçã 'Fuji', em relação à refrigeração. Nas condições brasileiras, esta cultivar deve ser armazenada em uma atmosfera com baixa pressão parcial de $\mathrm{CO}_{2}(<0,8$ $\mathrm{kPa}$ ) e com pressão parcial de $\mathrm{O}_{2}$ inferior a $1,5 \mathrm{kPa}$ (BRACKMANN et al., 1995). Condições inadequadas de AC promovem a incidência de distúrbios fisiológicos, principalmente de degenerescência da polpa, que é responsável por grandes perdas durante o armazenamento de maçãs 'Fuji'. Esse distúrbio se caracteriza por um escurecimento na região do córtex, que não atinge a região

\footnotetext{
${ }^{1}$ Engenheiro Agrônomo, Doutor em Ciências Agrárias - Professor Adjunto, Departamento de Fitotecnia -Universidade Federal de Santa Maria/UFSM 97105-900 - Santa Maria, RS - brackman@ccr.ufsm.br

2 Engenheiro Agrônomo, mestrando do Programa de Pós-Graduação em Agronomia/PPGA da UFSM - Bolsista CNPq - hetgiehI@yahoo.com.br

3 Engenheiro Agrônomo, mestrando do PPGA da UFSM - Bolsista CNPq - isestari@yahoo.com.br

${ }^{4}$ Engenheiro Agrônomo, Mestre em Agronomia, doutorando do PPGA da UFSM - Bolsista CAPES - cristianosteffens@bol.com.br
} 
carpelar. Várias são as causas relacionadas a esse distúrbio fisiológico durante o armazenamento, destacando-se a alta umidade relativa do ar, baixa temperatura de armazenamento (LITTLE \& BARRAND, 1989), alta incidência de pingode-mel (STAINER, 1990) e baixa pressão parcial de O ${ }_{2}$ (LAU et al., 1987). Para Peppelenbos \& Oosterhaven (1996), o dano causado em pressões parciais de $\mathrm{O}_{2}$ muito baixas é devido a níveis insuficientes de ATP, necessários para manter os requerimentos de energia. Com isso, os processos de reparação das membranas celulares cessam e as células morrem, causando escurecimento e necrose dos tecidos.

Técnicas complementares de AC, como a utilização de pressões parciais ultra-baixas de $\mathrm{O}_{2}$, situadas entre 0,5 $\mathrm{kPa}$ e $1,0 \mathrm{kPa}$ ("ultra low oxigen" - ULO), têm sido estudadas em muitas cultivares. A esse respeito, Curry (1989) não verificou a ocorrência de degenerescência da polpa em maçãs 'Fuji' armazenadas em AC com pressões parciais de $0,5 \mathrm{kPa}$ e $1,0 \mathrm{kPa}$ de $\mathrm{O}_{2}$. Já Bortoluzzi (1997) observou que a redução do $\mathrm{O}_{2}$ de $1,5 \mathrm{kPa}$ para $0,7 \mathrm{kPa}$ aumentou a incidência de degenerescência da polpa nessa cultivar, sendo observados sinais de fermentação nos frutos armazenados na última condição.

A umidade relativa do ar (UR) é outro fator muito importante no armazenamento. A alta UR predispõe os frutos à ocorrência de degenerescência (LITTLE \& BARRAND, 1989), ao ataque de fungos e a rachaduras (SCHWARZ, 1994). Brackmann et al. (1995) verificaram menor incidência de degenerescência na maçã 'Fuji' com a diminuição da UR de $97 \%$ para $75 \%$. No entanto, os frutos apresentaram-se desidratados ao final do período de armazenamento. De acordo com Werner (1989), o armazenamento dos frutos por um período curto em baixa UR possibilita uma regressão na severidade de pingo-demel, reduzindo futuras ocorrências de degenerescência da polpa em frutos com alta incidência desse distúrbio fisiológico.

Tendo em vista a existência de divergências quanto às melhores condições de armazenamento de maçãs 'Fuji', objetivou-se avaliar o efeito da temperatura, da umidade relativa, de pressões ultra-baixas de $\mathrm{O}_{2}$ e do retardamento do resfriamento e da instalação da atmosfera controlada sobre as qualidades físico-químicas dos frutos.

\section{MATERIALEMÉTODOS}

O experimento foi conduzido no Núcleo de Pesquisa em Pós-Colheita (NPP) do Departamento de Fitotecnia da Universidade Federal de Santa Maria, RS. Os frutos utilizados foram colhidos em um pomar comercial do município de Vacaria, RS. Ao chegarem ao NPP, os frutos foram selecionados e descartados os danificados e com podridões. Posteriormente, as amostras experimentais foram homogeneizadas. O delineamento experimental utilizado foi o inteiramente casualizado; com sete tratamentos com quatro repetições, sendo as unidades experimentais compostas por 25 frutos. As condições avaliadas foram: armazenamento a $0,5^{\circ} \mathrm{C}$ sob AC com (1) $0,7 \mathrm{kPa}$ de $\mathrm{O}_{2}$ e (2) $1,0 \mathrm{kPa}$ de $\mathrm{O}_{2}$; armazenamento $\mathrm{a}-0,5^{\circ} \mathrm{C}$ sob AC com (3) 0,7 kPa de $\mathrm{O}_{2}$; (4) 1,0 $\mathrm{kPa} \mathrm{de} \mathrm{O}_{2}$; (5) 1,0 kPa de $\mathrm{O}_{2}$ mais baixa UR na câmara; (6) $1,0 \mathrm{kPa}$ de $\mathrm{O}_{2}$, após 2 dias de exposição a $20^{\circ} \mathrm{Ce}$ (7) $1,0 \mathrm{kPa}$ de $\mathrm{O}_{2}$ após 1 mês de armazenamento refrigerado (AR).

Os frutos foram armazenados em minicâmaras experimentais com $80 \mathrm{~L}$ de volume, fechadas de forma hermética, nas quais instalou-se a condição de atmosfera referente a cada tratamento. Para a instalação da atmosfera, reduziu-se a pressão parcial de $\mathrm{O}_{2}$ com a injeção de nitrogênio produzido por um gerador de $\mathrm{N}_{2}$, que usa o princípio "Pressure Swing Adsorption" (PSA). Para evitar o acúmulo de $\mathrm{CO}_{2}$ utilizou-se cal hidratada no interior das minicâmaras. Para manter baixa a umidade relativa durante o armazenamento dos frutos submetidos à baixa UR (90\%), utilizou-se cloreto de cálcio $\left(\mathrm{CaCl}_{2}\right)$. Além disso, instalouse um psicrômetro no interior dessa minicâmara, para acompanhar a UR de modo a determinar o momento de se efetuar a troca do $\mathrm{CaCl}_{2}$, quando este saturava.

As minicâmaras de AC foram conectadas a um equipamento eletrônico de medição e controle automático de gases da marca Kronenberger, sendo realizadas as correções das pressões parciais dos gases diversas vezes ao dia. As temperaturas das câmaras frigoríficas foram reguladas automaticamente por meio de termostatos de alta precisão e acompanhadas diariamente por meio de termômetros com bulbo de mercúrio inseridos na polpa de alguns frutos.

Após oito meses foram realizadas as análises laboratoriais no momento da saída dos frutos das câmaras e após sete dias de exposição a $20^{\circ} \mathrm{C}$. Os parâmetros avaliados foram: cor de fundo da epiderme: determinada com o auxílio de um colorímetro da marca Minolta, que utiliza o sistema tridimensional $\mathrm{L}^{*} \mathrm{a} * \mathrm{~b} *$ de cores e os resultados expressos pela soma de $a^{*}+b^{*}$, sendo que quanto maior esse valor mais amarela e menos verde é a coloração de fundo da epiderme; firmeza da polpa: avaliada na região equatorial dos frutos, em lados opostos, com um penetrômetro manual com ponteira de $11,5 \mathrm{~mm}$ de diâmetro

Ciênc. agrotec., Lavras, v. 29, n. 4, p. 803-809, jul./ago., 2005 
e os resultados expressos em Newton (N); sólidos solúveis totais (SST): determinados com o auxílio de um refratômetro manual com correção da temperatura e expressos em ${ }^{\circ}$ Brix; acidez titulável: determinada pela titulação de uma solução contendo $10 \mathrm{~mL}$ de suco diluído em $100 \mathrm{~mL}$ de água destilada com $\mathrm{NaOH}(0,1 \mathrm{~N})$ até $\mathrm{pH} 8,1$, sendo os resultados expressos em meq $100 \mathrm{~mL}^{-1}$; degenerescência da polpa: avaliada pela contagem de frutos que apresentavam, internamente, sintomas característicos desse distúrbio fisiológico e os resultados expressos em porcentagem de frutos com esse distúrbio; incidência de podridões: determinada pela contagem dos frutos que apresentavam lesões com diâmetro superior a $5 \mathrm{~mm}$ e com sintomas característicos de ataques de fungos, sendo expressa em porcentagem de frutos podres.

Os dados, expressos em porcentagem, foram transformados pela fórmula $\operatorname{arc}$. sen $\sqrt{x / 100}$ antes de proceder a análise da variância. As médias foram comparadas entre si pelo teste de Duncan em nível de 5\% de probabilidade de erro.

\section{RESULTADOSE DISCUSSÃO}

Após oito meses de armazenamento, independente dos tratamentos, os frutos não apresentaram diferença significativa na firmeza da polpa, na saída da câmara (Tabela 1). Esses resultados concordam com Brackmann \& Saquet (1995), que não obtiveram diferenças significativas na firmeza da polpa de frutos armazenados em pressões parciais de $1,0 \mathrm{kPa}, 1,5 \mathrm{kPa}$ e $2,0 \mathrm{kPa}$ de $\mathrm{O}_{2}$ a $1{ }^{\circ} \mathrm{Ce} 2^{\circ} \mathrm{C}$. No entanto, após sete dias de exposição a $20^{\circ} \mathrm{C}$, firmeza da polpa mais elevada foi observada nos frutos submetidos à pressão parcial de $0,7 \mathrm{kPa}$ de $\mathrm{O}_{2}$ à temperatura de $-0,5^{\circ} \mathrm{C}$, diferindo estatisticamente apenas do tratamento em que as condições de $\mathrm{AC}$ foram instaladas após um mês de armazenamento refrigerado (Tabela 2). A retenção da firmeza da polpa em condições de oxigênio ultra-baixo deve-se à diminuição da respiração e conseqüente retardamento do amadurecimento. No entanto, Bortoluzzi (1997) não obteve diferença significativa na firmeza da polpa de maçãs 'Fuji' armazenadas a $0,7 \mathrm{kPa}$ e $1,0 \mathrm{kPa}$ de $\mathrm{O}_{2}$ nas temperaturas de 0 e $1,0^{\circ} \mathrm{C}$.

Os frutos armazenados a $-0,5^{\circ} \mathrm{C}$ e submetidos a 1,0 $\mathrm{kPa}$ de $\mathrm{O}_{2}$ e baixa UR (90\%) apresentaram a maior acidez titulável e os maiores teores de SST na saída da câmara (Tabela 1). Esses frutos estavam com características visuais de murchamento (dados não apresentados), em função da perda excessiva de água, o que deve ter concentrado os açúcares e os ácidos orgânicos no suco (BRACKMANN et al., 1999). Portanto, deve ser evitado o uso de baixa UR por tempo prolongado. O atraso no resfriamento também manteve teores mais elevados de SST, porém não diferindo dos frutos submetidos ao armazenamento a $0,5^{\circ} \mathrm{C}$ sob $\mathrm{AC}$ com $0,7 \mathrm{kPa}$ de $\mathrm{O}_{2}$ e ao atraso da instalação da AC (Tabela 1). Brackmann et al. (1998) não verificaram diferenças nos valores de SST em maçãs 'Fuji' em função do período de instalação da AC. No entanto, não há na literatura informações sobre o efeito do atraso do resfriamento sobre a manutenção dos teores de SST. Após sete dias de exposição a $20^{\circ} \mathrm{C}$, os teores de SST dos frutos foram iguais, estatisticamente, entre os tratamentos (Tabela 2), concordando com Bortoluzzi (1997) e Brackmann et al. (1998), que não verificaram efeito das condições de armazenamento sobre esse parâmetro em maçãs 'Fuji'. Já em relação à acidez titulável, os frutos submetidos à baixa UR apresentaram novamente os maiores valores absolutos para esse parâmetro, diferindo estatisticamente apenas dos frutos submetidos a 1,0 $\mathrm{kPa}$ de $\mathrm{O}_{2}$ - após 1 mês em AR (Tabela 2). $\mathrm{O}$ atraso na instalação da $\mathrm{AC}$ promoveu uma redução mais pronunciada da acidez titulável, provavelmente pelo consumo mais acentuado de ácidos orgânicos durante o período em que os frutos permaneceram somente em condições refrigeradas.

Os frutos armazenados $\mathrm{a}-0,5^{\circ} \mathrm{C}$ sob $\mathrm{AC}$ com 0,7 $\mathrm{kPa}$ e 1,0 kPa de $\mathrm{O}_{2}$ apresentaram epiderme mais verde e menos amarela, na saída da câmara, em comparação aos frutos submetidos a essas mesmas condições de $\mathrm{AC}$ a $0,5^{\circ} \mathrm{C}$ e ao uso de baixa UR (Tabela 1). Além disso, os frutos submetidos ao atraso do resfriamento e da instalação da AC, estavam mais verdes e menos amarelos que os submetidos à baixa UR (Tabela 1), sugerindo que a desidratação dos frutos pode ter acarretado um aumento no metabolismo ou na síntese de etileno, pois Nakano et al. (2003) observaram que a perda de água, em caquis colhidos imaturos, estimulou a biossíntese de etileno. Para Sharples (1982), a menor perda da cor verde da epiderme pode ser obtida com o uso de baixas pressões de $\mathrm{O}_{2}$, pela inibição da degradação das clorofilas. Após sete dias de exposição a $20^{\circ} \mathrm{C}$, os frutos submetidos a $1,0 \mathrm{kPa}$ de $\mathrm{O}_{2}$ à temperatura de $0,5^{\circ} \mathrm{C}$ apresentavam a epiderme mais verde e menos amarela, quando comparados a essa mesma pressão parcial de $\mathrm{O}_{2} \mathrm{a}-0,5^{\circ} \mathrm{C}$ (Tabela 2). 
TABELA 1 - Qualidade de maçãs ‘Fuji’ em função da temperatura, pressões parciais de $\mathrm{O}_{2}$, UR e retardamento da refrigeração e instalação da atmosfera contolada após 8 meses armazenamento, Santa Maria, RS.

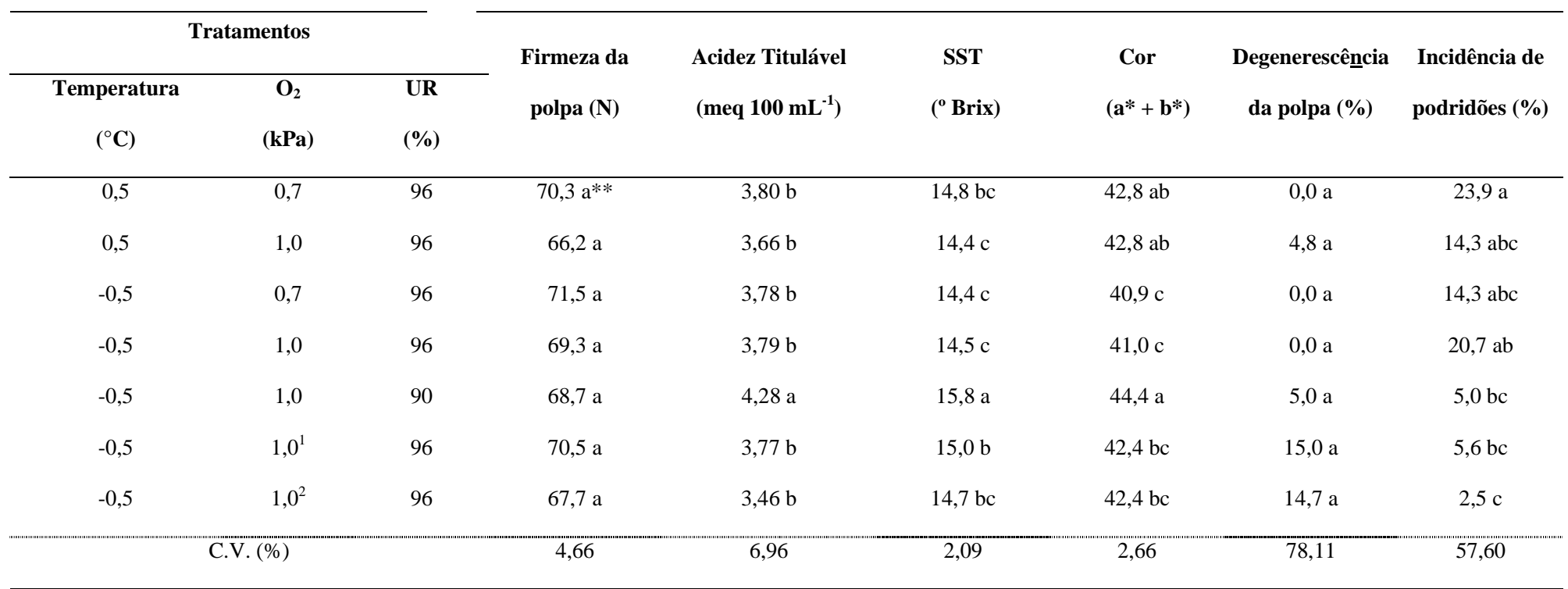

${ }^{1}$ Os frutos foram submetidos por dois dias a $20^{\circ} \mathrm{C}$ antes do resfriamento;

${ }^{2}$ Os frutos foram submetidos por um mês ao armazenamento refrigerado antes da instalação da AC.

* As pressões parciais de $\mathrm{CO}_{2}$ foram mantidas abaixo de $0,5 \mathrm{kPa}$;

** Médias seguidas pela mesma letra não diferem estatisticamente entre si pelo teste de Duncan em nível de $5 \%$ de probabilidade de erro. 
TABELA 2 - Qualidade de maçãs 'Fuji' em função da temperatura, pressões parciais de $\mathrm{O}_{2}$, UR e retardamento da refrigeração e instalação da atmosfera controlada após 8 meses armazenamento mais sete dias a $20^{\circ} \mathrm{C}$. Santa Maria, RS.

\begin{tabular}{|c|c|c|c|c|c|c|c|c|}
\hline \multicolumn{3}{|c|}{ Tratamentos } & \multirow{2}{*}{$\begin{array}{c}\text { Firmeza da } \\
\text { polpa }(\mathbf{N})\end{array}$} & \multirow{2}{*}{$\begin{array}{l}\text { Acidez Titulável } \\
\text { (meq } 100 \text { mL }^{-1} \text { ) }\end{array}$} & \multirow{2}{*}{$\begin{array}{l}\text { SST } \\
\left({ }^{\circ} \text { Brix }\right)\end{array}$} & \multirow{2}{*}{$\begin{array}{l}\text { Cor } \\
\left(a^{*}+b^{*}\right)\end{array}$} & \multirow{2}{*}{$\begin{array}{c}\text { Degenerescência } \\
\text { da polpa (\%) }\end{array}$} & \multirow{2}{*}{$\begin{array}{l}\text { Incidência de } \\
\text { podridões (\%) }\end{array}$} \\
\hline $\begin{array}{c}\text { Temperatura } \\
\left({ }^{\circ} \mathrm{C}\right)\end{array}$ & $\begin{array}{c}\mathrm{O}_{2} \\
(\mathbf{k P a})\end{array}$ & $\begin{array}{l}\text { UR } \\
(\%)\end{array}$ & & & & & & \\
\hline 0,5 & 0,7 & 96 & $68,4 \mathrm{ab}^{* *}$ & $3,71 \mathrm{ab}$ & $14,8 \mathrm{a}$ & $44,0 \mathrm{ab}$ & $5,3 a b$ & $75,0 \mathrm{a}$ \\
\hline 0,5 & 1,0 & 96 & $68,4 \mathrm{ab}$ & $3,67 \mathrm{ab}$ & $15,2 \mathrm{a}$ & $41,6 \mathrm{~b}$ & $6,7 \mathrm{ab}$ & $97,2 \mathrm{a}$ \\
\hline$-0,5$ & 0,7 & 96 & $69,1 \mathrm{a}$ & $3,75 \mathrm{ab}$ & $15,1 \mathrm{a}$ & $44,2 \mathrm{ab}$ & $2,5 \mathrm{~b}$ & $45,0 \mathrm{ab}$ \\
\hline$-0,5$ & 1,0 & 96 & $67,8 \mathrm{ab}$ & $3,60 \mathrm{ab}$ & $14,9 \mathrm{a}$ & $45,4 \mathrm{a}$ & $12,3 \mathrm{a}$ & $41,8 \mathrm{ab}$ \\
\hline$-0,5$ & 1,0 & 90 & $66,2 a b$ & $3,98 \mathrm{a}$ & $15,3 \mathrm{a}$ & $45,2 \mathrm{ab}$ & $5,0 \mathrm{ab}$ & $55,0 \mathrm{ab}$ \\
\hline$-0,5$ & $1,0^{1}$ & 96 & $66,1 \mathrm{ab}$ & $3,67 \mathrm{ab}$ & $15,2 \mathrm{a}$ & $42,4 \mathrm{ab}$ & $7,5 \mathrm{ab}$ & $22,5 \mathrm{~b}$ \\
\hline$-0,5$ & $1,0^{2}$ & 96 & $65,4 \mathrm{~b}$ & $3,31 \mathrm{~b}$ & $14,9 \mathrm{a}$ & $44,2 \mathrm{ab}$ & $12,5 \mathrm{a}$ & $35,0 \mathrm{~b}$ \\
\hline & V. $(\%)$ & & 3,23 & 7,73 & 2,69 & 5,08 & 69,65 & 48,85 \\
\hline
\end{tabular}

${ }^{1}$ Os frutos foram submetidos por dois dias a $20^{\circ} \mathrm{C}$ antes do resfriamento;

${ }^{2}$ Os frutos foram submetidos por um mês ao armazenamento refrigerado antes da instalação da AC

* As pressões parciais de $\mathrm{CO}_{2}$ foram mantidas abaixo de $0,5 \mathrm{kPa}$;

**Médias seguidas pela mesma letra não diferem estatisticamente entre si pelo teste de Duncan em nível de 5\% de probabilidade de erro. 
Em relação à degenerescência da polpa, não se constatou diferença significativa entre os frutos dos tratamentos testados, na saída da câmara (Tabela 1). O uso de baixa UR não foi eficiente no controle da degenerescência de polpa, contrariando Little \& Barrand (1989). Segundo esses autores, o uso de umidade relativa elevada promove o desenvolvimento de degenerescência da polpa. Quanto ao uso de temperatura mais elevada no início do armazenamento, Little \& Peggie (1987) observaram uma menor incidência de degenerescência da polpa em maçãs 'Jonathan' e 'Granny Smith', com a redução gradativa da temperatura de armazenamento até $0{ }^{\circ} \mathrm{C}$. De acordo com Little \& Barrand (1989), a susceptibilidade de determinadas frutas à baixa temperatura pode ser diminuída mediante resfriamento gradativo, pois o estresse por baixa temperatura é mais crítico no início do armazenamento. Após sete dias a $20^{\circ} \mathrm{C}$, os frutos armazenados à temperatura de $-0,5^{\circ} \mathrm{C}$ sob AC com $0,7 \mathrm{kPa}$ de $\mathrm{O}_{2}$ apresentaram a menor incidência de degenerescência da polpa, especialmente quando comparados aos frutos submetidos ao atraso na instalação da $\mathrm{AC}$ e ao uso de $1,0 \mathrm{kPa}$ de $\mathrm{O}_{2}$ a $-0,5^{\circ} \mathrm{C}$ (Tabela 2). Curry (1989) não observou variação na incidência de degenerescência da polpa em maçãs 'Fuji' com o abaixamento das pressões parciais de $\mathrm{O}_{2}$ para 0,5 $\mathrm{kPa}$. Também Bortoluzzi (1997) não encontrou diferença significativa entre frutos armazenados sob 1,0 e $0,7 \mathrm{kPa}$ de $\mathrm{O}_{2}$, quanto à incidência desse distúrbio.

A incidência de podridões, na saída da câmara, foi menor nos frutos mantidos em AR durante um mês antes da instalação da $\mathrm{AC}$ com $1,0 \mathrm{kPa}$ de $\mathrm{O}_{2}$, diferindo estatisticamente apenas do tratamento com $0,7 \mathrm{kPa}$ de $\mathrm{O}_{2}$ a $0,5^{\circ} \mathrm{Ce} 1,0 \mathrm{kPa}$ de $\mathrm{O}_{2} \mathrm{a}-0,5^{\circ} \mathrm{C}$ (Tabela 1 ). O uso de baixa UR e o atraso no resfriamento também apresentaram bons resultados nesse parâmetro, determinando menor incidência de podridões em comparação à $\mathrm{AC}$ com $0,7 \mathrm{kPa}$ de $\mathrm{O}_{2}$ a $0,5^{\circ} \mathrm{C}$. De acordo com Schwarz (1994), a elevada incidência de podridões está relacionada à alta UR durante o armazenamento. No entanto, Brackmann et al. (1995) não obtiveram efeito significativo com o uso de baixa UR e de alta temperatura no início do armazenamento sobre o controle de podridões em maçãs 'Fuji'. Após sete dias à temperatura de $20^{\circ} \mathrm{C}$, os frutos submetidos ao retardamento do resfriamento e ao atraso na instalação da $\mathrm{AC}$, apresentaram menor ocorrência de podridões, diferindo estatisticamente dos frutos armazenados em $\mathrm{AC}$ a $0,5^{\circ} \mathrm{C}$ (Tabela 2).

\section{CONCLUSÕES}

De modo geral, as condições de armazenamento avaliadas neste trabalho não proporcionaram diferenças significativas na qualidade de maçãs 'Fuji', após oito meses de armazenamento e mais sete dias de exposição a $20^{\circ} \mathrm{C}$. No entanto, o atraso no resfriamento, por meio da exposição dos frutos a $20^{\circ} \mathrm{C}$ por dois dias antes do armazenamento a $-0,5^{\circ} \mathrm{C}$ sob AC com $1,0 \mathrm{kPa}$ de $\mathrm{O}_{2}+<0,5 \mathrm{kPa}$ de $\mathrm{CO}_{2}$, e o retardamento na instalação das condições de $\mathrm{AC}(1,0 \mathrm{kPa}$ de $\mathrm{O}_{2}+<0,5 \mathrm{kPa}$ de $\mathrm{CO}_{2} \mathrm{a}-0,5^{\circ} \mathrm{C}$ ) em um mês, apresentaram bons resultados no controle das podridões após sete dias a $20^{\circ} \mathrm{C}$, especialmente quando comparados com o armazenamento a $0,5^{\circ} \mathrm{C}$ sob AC com 0,7 e $1,0 \mathrm{kPa}$ de $\mathrm{O}_{2}$ $+<0,5 \mathrm{kPa}$ de $\mathrm{CO}_{2}$.

\section{REFERÊNCIAS BIBLIOGRÁFICAS}

BORTOLUZZI, G. Efeito das temperaturas de armazenamento e condições de atmosfera controlada sobre a qualidade da maçã 'Fuji'. 1997. 93 f. Dissertação (Mestrado em Agronomia) - Universidade Federal de Santa Maria, Santa Maria, 1997.

BRACKMANN, A.; BORTOLUZ, L.; BORTOLUZZI, G. Frigoconservação de maçã 'Fuji' em duas temperaturas e em atmosfera controlada. Revista Brasileira de Agrociência, Pelotas, v. 4, n. 1, p. 26-30, 1998.

BRACKMANN, A.; BORTOLUZZI, G.; BORTOLUZ, L. Controle da degenerescência da polpa da maçã 'Fuji' com concentrações dinâmicas de $\mathrm{O}_{2}$ e $\mathrm{CO}_{2}$ e redução da umidade relativa durante o armazenamento em atmosfera controlada. Ciência Rural, Santa Maria, v. 29, n. 3, p. 459-463, 1999.

BRACKMANN, A.; MAZARO, S. M.; BORTOLUZZI, G. Qualidade da maçã 'Fuji’ sob condições de atmosfera controlada. Ciência Rural, Santa Maria, v. 25, n. 2, p. 215-218, 1995.

BRACKMANN, A.; SAQUET, A. A. Efeito das condições de atmosfera controlada sobre a ocorrência de degenerescência em maçã 'Fuji'. Scientia Agricola, Piracicaba, v. 52, n. 2, p. 263-267, 1995.

CURRY, E. A. Effect of harvest date and oxygen level on storability of late season apple cultivars. In: INTERNATIONAL CONTROLLED ATMOSPHERE RESEARCH CONFENRENCE, 5., 1989, Washington. Proceedings... Washington: [s.n.], 1989. p. 103-109.

Ciênc. agrotec., Lavras, v. 29, n. 4, p. 803-809, jul./ago., 2005 
LAU, O. L.; YASTREMSKI, R.; MEHERIUK, M. Influence of maturity, storage procedure, temperature and oxygen concentration on quality and disorders of 'McIntosh' apples. Journal of the American Society for Horticultural Science, Alexandria, v. 111, n. 6, p. 93-99, 1987.

LITTLE, C. R.; BARRAND, L. The effect of preharvest, postharvest and storage conditions on some fruit disorders. In: INTERNATIONAL CONTROLLED ATMOSPHERE RESEARCH CONFERENCE, 5., 1989, Washington. Proceedings... Washington: [s.n.], 1989. p. 185-192.

LITTLE, C. R.; PEGGIE, I. D. Storage injury of pome fruit caused by stress levels of oxygen, carbon dioxide, temperature and ethylene. HortScience, Alexandria, v. 25, n. 5, p. 783-790, 1987.

NAKANO, R.; OGURA, E.; KUBO, I.; INABA, A. Ethylene biosynthesis in detached young persimmon is initiated in calix and modulated by water loss from the fruit. Plant Physiology, Rockville, v. 131, p. 276286, 2003.
PEPPELENBOS, H. W.; OOSTERHAVEN, J. A theorical approach on the role of fermentation in fruits and vegetables. In: INTERNATIONAL POSTHARVEST SCIENCECONFERENCE, 1996, Taupo. Abstracts... Taupo: [s.n.], 1996. p. 99.

SCWARZ, A. Relative humidity in cool stores: measurement control and influence of discreet factors. Acta Horticulturae, Wageningen, n. 368, p. 867-892, 1994.

SHARPLES, R. O. Effects of ultra-low oxygen conditions on the storage quality of English 'Cox's Orange Pippin' apples. In: SYMPOSIUM SERIES, 1982, Oregon. Proceedings... Oregon: Oregon State University-School of Agriculture, 1982.

STAINER, R. Gala, Braeburn und Fuji. Obstbau-Weinbau, Bonn, v. 2, p. 40-42, 1990.

WERNER, R. A. Current status of controlled atmosphere storage in Brazil. In: INTERNATIONAL CONTROLLED ATMOSPHERE RESEARCHCONFERENCE, 5., Wenatchee, 1989. Proceedings... Wenatchee: [s.n.], 1989. p. 509-515. 